\title{
Proust nose best: Odors are better cues of autobiographical memory
}

\author{
SIMON CHU and JOHN J. DOWNES \\ University of Liverpool, Liverpool, England
}

\begin{abstract}
The Proust phenomenon is an enduring piece of folk wisdom that asserts that odors are particularly powerful autobiographical memory cues. We provide a more formal exposition of this phenomenon and test it in two experiments, using a novel double-cuing methodology designed to negate less interesting explanations. In both studies, recall of an autobiographical event was initially cued by a verbal label (an odor name) for a fixed period, following which a second, extended recall attempt was cued by the same verbal label, the relevant odor, an irrelevant odor, or a visual cue. The focus of Experiment 1 was participants' ratings of the emotional quality of their autobiographical memories. In Experiment 2, content analysis was employed to determine the quantity of information in participants' recollections. Results revealed that odor-cued autobiographical memories were reliably different in terms of qualitative ratings and reliably superior in the amount of detail yielded. Moreover, visual cues and incongruent olfactory cues appeared to have a detrimental effect on the amount of detail recalled. These results support the proposal that odors are especially effective as reminders of past experience.
\end{abstract}

The belief that odors are especially evocative reminders of past experiences can be traced to the literary anecdote described by Marcel Proust, in which he was vividly reminded of his childhood when he dipped a madeleine biscuit into his tea (Proust, 1922/1960). The so-called Proust phenomenon has been defined more formally in a number of different ways (see Chu \& Downes, 2000). These vary from the belief that odors themselves are not forgotten as rapidly as other perceptual events (Engen \& Ross, 1973), to the belief that odors evoke older memories than do other stimuli (Rubin, Groth, \& Goldsmith, 1984), to the belief that odors evoke recollections that are more emotionally loaded (Herz \& Cupchik, 1995).

Engen and Ross (1973), for example, observed that whereas memory for odors, relative to stimuli from other perceptual modalities, was initially poor, subsequent testing revealed very little forgetting of the odors. This led them to conjecture that memory for odors differs in important ways from that for stimuli from other modalities, a view that has since been corroborated by a sizeable body of empirical evidence (for reviews, see Herz \& Engen, 1996; Schab, 1991; White, 1998) and that has led to the stronger claims that a separable memory system for olfaction exists and even that odor memory itself may dissociate into different

This research was supported by Unilever Research and by an Economic and Social Research Council studentship award to S.C. Thanks are extended to Richard Cotler, Daniel Farrelly, Alison Gall, Rosie Hales, Emily Highfield, and Kevin Haigh for assistance in data collection. We also thank ChristopherD. B. Burt, David C. Rubin, and an anonymous reviewer for helpful critiques on an earlier draft. Correspondence concerning this article should be addressed to J. J. Downes, Department of Psychology, University of Liverpool, Eleanor Rathbone Building, Bedford Street South, Liverpool L69 7ZA, England (e-mail: down@ liverpool.ac.uk). component subsystems (Lehrner, Walla, Laska, \& Deecke, 1999). Findings concerning the relative persistence of odors in memory are clearly important to proponents of Proustian retrieval because, for a stimulus to be an effective retrieval cue, it must first be present as part of the memory trace. However, the development of testable hypotheses about the Proustian phenomenon will necessarily involve more detailed consideration of the unique ways in which odors interact with other event information to produce mnemonic representations that are more detailed, more stable, and more emotionally loaded.

There have been surprisingly few attempts at investigating the Proust phenomenon. Some of these have examined experimentally induced memories and have reported them to be significantly more emotionally toned when cued by an odor, as compared with the odor's verbal label (Herz \& Cupchik, 1995) or the sight or touch of the source object (Herz, 1996). In other studies, naturally occurring autobiographical memories have been examined, and these are, therefore, of more direct relevance to the Proust phenomenon. Herz and Cupchik (1992) concluded that odor-evoked memories tended to be highly emotional, vivid, specific, rare, and relatively old. However, the main purpose of that study was to determine whether sex differences were evident in these qualitative aspects of autobiographical memory retrieval, and odors were not compared with other types of sensory cues. This comparison was made by Rubin et al. (1984), who gave odors, verbal labels, or photographs to participants and asked them to briefly describe and rate the retrieved autobiographical memories. Compared with the other cue types, odor-cued memories were rated as more pleasant and were thought of and spoken of less often.

Rubin et al.'s (1984) study highlights the obvious methodological approach to evaluating hypotheses that claim that autobiographical memories cued by odors differ in impor- 
tant ways from those cued by other sensory cues, an approach we refer to as the single cue comparisonmethod. The inclusion of a verbal label condition is important, because it allows one to evaluate an alternative, verbal mediation hypothesis. According to this, it is the generated odor name, rather than the odor itself, that operates as the retrieval cue. Finding that odor cues are significantly different on some critical dimension(s) from labels and from other types of sensory cue would therefore both negate this less interesting hypothesis and provide support for the Proust phenomenon. Although often not made explicit, the phenomenon has been commonly interpreted as a retrieval effect. A more formal version of this, which we refer to as the differential cue affordance hypothesis, is as follows. Cues of many different types become associated with autobiographical memories but may differ in terms of their associated affordance value, a hypothetical measure of how efficiently event details can be accessed. Proustian phenomena are explained by the higher cue affordance values of olfactory cues, as compared with other cue types.

It must be realized, however, that other interpretations are possible for the finding that odors are better cues than are stimuli from other sensory modalities. In contrast to the latter, retrieval-based hypothesis, factors operating at encoding may be important in producing the effect. One possibility, which we refer to as the differential encoding bias hypothesis, is as follows. Autobiographicalmemories differ in terms of the complexity of the underlying representations - that is, the number of event details that have been encoded and consolidated. However, the more complex a particular memory representation is, the greater the likelihood that peripheral details were initially encoded and consolidated. Olfactory details, then, which are peripheral in the sense that they do not usually alter the meaning or interpretation of an event (see Baddeley, 1982), may tend to be encoded only with more complex autobiographical memories. It follows that, other things being equal, olfactory cues will lead to the recovery of more detailed autobiographical memories than will other cue types.

Thus, although the accepted interpretation of the Proust phenomenon is that smells are better cues, a plausible alternative exists. Obviously, with the single-cue comparison method, it is not possible to discriminate between these two alternatives. The main aim of the present investigation is to provide unambiguous evidence by which the differential cue affordance hypothesis can be judged. To achieve this aim, it becomes necessary to compare different cues anchored to the same autobiographicalevent, and we will describe a methodology that we believe accomplishes this.

In the present study, rather than comparing different single cues, a double-cuing methodology is adopted (see Tulving \& Bower, 1974, for a similar approach). Thus, all autobiographical episodes are initially retrieved in response to a verbal label (an odor name), but this is followed by an extended retrieval prompted by a second cue, which can be the odor (corresponding to the verbal label), a lit- eral repetition (the verbal label), or, in Experiment 2, a conceptual repetition (a picture). In this way, in the first phase, participants' retrieval attempts are anchored to specific autobiographical episodes, using a label, and the relative effectiveness of cues from different sensory modalities can be evaluated in the second retrieval phase. The value of this approach is that any advantage observed for odors cannot be due to a differential encoding bias, because for this to operate would require that the odors, rather than their corresponding labels, initially cue the autobiographical episodes. In the experiments that follow, we do not sample all possible cue combinations. Consideration of these other conditions may help in evaluating more complex hypotheses about the relative contributions of encoding and retrieval factors in odor-cued autobiographical memory. However, our remit for the present work was more limited, and we included only those cue combinations that were minimally required to evaluate the differential cue affordance hypothesis.

The hypotheses just described do not exhaust the mechanisms that potentially operate in influencing the retrieval and/or interpretation of odor-cued memories. A brief discussion of some of these, and how they can be controlled for, is particularly important for Experiment 1, which focuses on the emotional aspects of retrieved memories. One problem relates to the fact that the hedonic valence of an odor is known to influence emotion (Baron \& Bronfen, 1994; Ehrlichman \& Bastone, 1992; Ehrlichman \& Halpern, 1988; Lorig \& Schwartz, 1988; Roberts \& Williams, 1992), a factor that may, in turn, determine either the actual or the perceived emotional valence of retrieved episodes. Thus, mood-congruent retrieval effects (e.g., Clark \& Teasdale, 1982), whereby the hedonic tone of retrieved memories matches that induced by the odor, may operate. Under the double-cuing methodology, however, this particular type of biased retrieval cannot operate, because the same cue type (label) is used for the initial retrieval of an episode across all conditions. A second type of bias might occur if the interpretation of emotion (believed to be) experienced during a past episode was influenced by the emotional state induced by the odor at the time of retrieval (see Levine, 1997), a kind of state-dependent effect. It is this type of explanation that Herz and Cupchik (1995) seem to invoke when discussing their finding that odor cues led to memory retrievals that were more emotionally toned. The contribution of this potential bias can be evaluated by the use of an additional control condition, in which a verbal label is paired with an incongruent odor. If this bias operates, the effect should emerge irrespective of the congruence between label and odor, thereby allowing one to discriminate between it and the differential cue affordance hypothesis

In what follows, two experiments in which the doublecuing methodology was employed are described. The first of these is concerned with memory for the emotional aspects of retrieved autobiographical episodes, and the second emphasizes the total amount of event detail recovered. 


\section{EXPERIMENT 1}

\section{Method}

Participants. Forty-two volunteer participants were drawn equally from the undergraduate population at Liverpool University and people in full-time employment known to the experimenters.

Materials. Ten odors were chosen on the basis of familiarity and ease of procurement. These were coffee, vinegar, ginger, chocolate, whiskey, onion, Parmesan cheese, peanut, cinnamon, and lemon. A small amount of each substance was placed in a wrapped glass jar, with a punctured lid to allow participants to smell the contents without the substance's being visible. The contents of the jars were replaced regularly to maintain odor quality and freshness.

Seven rating scales were constructed to measure the participants' evaluations of recalled memories on a selection of dimensions. Each scale was a 7-point Likert scale, and the selection of targeted dimensions was determined primarily by previously published work (Rubin et al., 1984). The dimensions were pleasant, painful, embarrassing, anxious, vivid, unique, and personally significant. For each dimension, the participants were asked the following: "on a scale of 1-7, how [pleasant/painful/etc.] are the events in this memory, where 1 is not [pleasant/painful/etc.] at all and 7 is extremely [pleasant/ painful/etc.]."

Design. A mixed design was used. All the participants were cued twice, with the first cue always being the verbal label. After each retrieval attempt, the participants completed the seven rating scales in the same fixed order. The second cue was either the (repeated) verbal label or an odor, and the odor was either of the same nominal identity as the first (verbal label) cue or different. The three levels of cue combination-label, congruent odor, and incongruent odor-made up the between-subjects factor of the design. In other words, the participants always received the same cue combinations. The 10 odors were divided into two stimulus sets of 5 each, and the participants were presented with labels from only one of the sets, with the other set forming the alternate odors for the incongruent odor condition. Stimulus sets were used equally often across participants and groups.

Procedure. The participants were tested in quiet distraction-free surroundings, in which there were no strong odors. All the participants were provided with the following instructions:

This is a study investigating memory for life events of the past. I am now going to give you a word. I would like you to relate an event in your past history which you associate with that word. When you do, please give as much detail about the event as you can remember. Please restrict yourself to a single event and not a series of events which occurred around the same time.

After this, an odor label was presented auditorily, and the participant was allowed 3 min to think of and relate verbally an autobiographical memory cued by the label, following which the rating scales were completed. No record was made of the participants' accounts of their memories. The second cue (label or odor) was then introduced with the following instructions.

Label group: "It has been suggested that additional effort may be a factor in improving memory performance, and so, given the word I would like you to try as hard as you can for a further few minutes to remember anything else about that event that you have just described."

Congruent and incongruent odor groups: "It has been suggested that additional effort may be a factor in improving memory performance and that an odor might stimulate memory and help remember details about past events. Here is an odor ... sniff at it and try as hard as you can for a further few minutes to remember anything else about the event you have just described." One reviewer queried whether the additional instruction, which stated that "odors might stimulate memory and help remember details about past events," used in the odor conditions, might have induced additional motiva- tion to retrieve detail. Furthermore, this motivation would have been diluted in the incongruent cue condition when participants perceived the mismatch between the cue and the retrieved episode. We believe it unlikely that these differences influenced the resulting data. The participants were not aware of the design or nature of the study, and a participant's perception of a mismatch between odor and retrieved episode should not have reduced their motivation to retrieve event detail. In addition, the participants had already been told that "additional effort may be a factor in improving memory performance," and the specific mention of "detail" in the odor conditions should not have served to dramatically influence responses.

The participants were given a further 3 min on this task, following which they completed the ratings scales again. This procedure was repeated for five trials, using different verbal cues on each trial. At the end of the study, all the participants were fully debriefed.

\section{Results and Discussion}

The data from the rating scales were transformed by calculating the change in ratings between the first and the second cues. For each participant, change scores were averaged across the five trials to give a single mean change score for each scale. Since it was not possible to specify a priori the direction of change for five of the scales (pleasant, personal, painful, embarrassing, and anxious), the absolute change on these scales served as the unit of analysis. If congruent odor cues afford access to a more vivid record of the emotional experiences associated with a retrieved episode, it would be equally likely that, for example, pleasantness would be reevaluated more or less pleasantly than beforehand. The actual direction would depend on the event that was retrieved, something over which we had no control. For the remaining two scales (vivid and unique), direction of change could be specified a priori, because access to a more detailed record of the emotion experienced during an event should render the episode more vivid and more unique. Thus, for these two scales, the prediction was that significant changes would occur in the positive direction.

Mean initial and subsequent ratings for each scale in each condition are shown in Table 1, from which it is apparent that memories were rated as being low on the painful, anxiety, and embarrassing scales with a large degree of consistency across all three conditions, whereas the vividness of the memories was reported to be quite high. Mean changes in ratings for each scale in each condition are also shown in Table 1, where it is clear that the changes in ratings in the congruent cue condition were greater than those in the other two conditions. Significantly, on the scales on which a direction of change could be specified a priori, the changes were both in the correct direction.

Owing to a strong positive skew, the data for each scale were analyzed, using nonparametric Kruskal-Wallis oneway analyses of variance (ANOVAs), with group as the between-subjects factor. Since nonparametric analyses were used, median changes in ratings for each of the scales are also shown in Table 1 . These analyses revealed significant main effects for six of the seven scales [for all reported statistics, $d f=2$; anxious, $\chi^{2}=13.11, p=.0014$; embarrassing, $\chi^{2}=7.91, p=.0192$; painful, $\chi^{2}=19.32$, 
$p<.001$; personal, $\chi^{2}=8.78, p=.0124$; pleasant, $\chi^{2}=$ $17.52, p<.001$; vivid, $\left.\chi^{2}=13.08, p=.0014\right]$. Analyses were also carried out with parametric tests (ANOVAs), and revealed a very similar pattern of results, with the exception that between-groups differences on the vivid scale only reached borderline significance $(p=.065)$.

Post hoc multiple comparisons between groups (Siegel \& Castellan, 1988) showed that the mean ranks of change scores for the label group were no different from those for the incongruent odor group on all but the painful scale, for which the mean rank for label group was significantly lower (where smallest changes rank highest). More important, the mean of the change score ranks for the congruent odor group were significantly lower than those for the incongruent odor group on all the scales and were significantly lower than those for the label group on four (pleasant, anxious, painful, and vivid) of the six scales for which there was a significant main effect. Furthermore, in the case of the vivid scale, the change was in the predicted direction, so that retrieved memories were rated as more vivid in the congruent odor condition. On only one scale did the congruent odor group not show a significantly lower mean rank [unique, $\chi^{2}=4.95, p=.0842$ ] .

All the shifts in ratings in the incongruent cue and label cue conditions were small or negligible, but one interesting result was the negative shift in the unique ratings in the label condition. Encouragement to retrieve additional details concerning the remembered episode in the label condition, when little or no event-specific detail is forth- coming, may only serve to yield more nonspecific, schemabased knowledge. The activation of such knowledge might serve to alter the perception of the remembered episode, so that it would appear less unique in the light of schematically similar episodes. (D. C. Rubin, personal communication).

As was noted earlier, use of the single-cue comparison method could mean that any difference between olfactory and other cues resulted from the retrieval of more complex memories, as is postulated by the differential encoding bias hypothesis. However, because direct cuing is prevented by the double-cuing methodology, we can be confident that the results obtained reflected the fact that odors are more potent retrieval cues. A similar argument applies to mood-congruity retrieval effects. Finally, the fact that a similar effect was not observed for the incongruent odor condition rules out another alternative hypothesis, that odors bias participants' responses as a result of the emotional state they tend to induce. Thus, the effects observed are memory specific. The present results therefore provide the first unambiguous evidence supporting one popular interpretation of the Proust phenomenon, that odors are especially evocative as retrieval cues for the emotional details of autobiographical memory episodes.

Four of the scales revealed greater changes in ratings in the congruent odor group, but there are grounds for believing that the two scales for which the pattern was less clear-embarrassing and personal — may be, by their very nature, less valid probes for emotionality. In real life, em-

Table 1

Mean Initial (R1) and Subsequent (R2) Ratings, Mean Change in Ratings (With Standard Deviations), and Median Change in Ratings for Each Scale in Each Condition

\begin{tabular}{|c|c|c|c|c|c|c|c|}
\hline & \multicolumn{2}{|c|}{$R 1$} & \multicolumn{2}{|c|}{$R 2$} & \multicolumn{2}{|c|}{ Change } & \multirow{2}{*}{$\begin{array}{l}\text { Median } \\
\text { Change }\end{array}$} \\
\hline & $M$ & $S D$ & $M$ & $S D$ & $M$ & $S D$ & \\
\hline \multicolumn{8}{|c|}{ Incongruent Cue Condition } \\
\hline Pleasant & 4.04 & 2.08 & 4.03 & 2.07 & 0.07 & 0.16 & 0 \\
\hline Personal & 2.54 & 1.90 & 2.53 & 1.88 & 0 & 0 & 0 \\
\hline Painful & 1.56 & 1.33 & 1.56 & 1.33 & 0 & 0 & 0 \\
\hline Anxious & 1.47 & 1.28 & 1.47 & 1.28 & 0 & 0 & 0 \\
\hline Embarrassing & 1.34 & 1.27 & 1.36 & 1.27 & 0.01 & 0.05 & 0 \\
\hline Vivid & 4.50 & 1.82 & 4.50 & 1.82 & 0 & 0 & 0 \\
\hline Unique & 3.31 & 1.65 & 3.21 & 1.62 & -0.10 & 0.17 & 0 \\
\hline \multicolumn{8}{|c|}{ Label Cue Condition } \\
\hline Pleasant & 3.24 & 3.00 & 3.23 & 3.00 & 0.16 & 0.21 & 0 \\
\hline Personal & 2.56 & 2.00 & 2.46 & 1.74 & 0.19 & 0.23 & 0.10 \\
\hline Painful & 1.56 & 1.09 & 1.60 & 1.18 & 0.07 & 0.13 & 0 \\
\hline Anxious & 1.77 & 1.28 & 1.79 & 1.31 & 0.04 & 0.08 & 0 \\
\hline Embarrassing & 1.57 & 1.25 & 1.51 & 1.15 & 0.06 & 0.14 & 0 \\
\hline Vivid & 5.14 & 1.63 & 5.11 & 1.66 & -0.03 & 0.07 & 0 \\
\hline Unique & 3.93 & 2.07 & 3.73 & 2.01 & -0.20 & 0.19 & -0.20 \\
\hline \multicolumn{8}{|c|}{ Congruent Cue Condition } \\
\hline Pleasant & 4.13 & 1.74 & 4.20 & 1.86 & 0.47 & 0.34 & 0.40 \\
\hline Personal & 3.07 & 2.07 & 3.06 & 2.12 & 0.48 & 0.50 & 0.22 \\
\hline Painful & 1.84 & 1.45 & 1.97 & 1.65 & 0.44 & 0.50 & 0.20 \\
\hline Anxious & 1.99 & 1.64 & 1.96 & 1.65 & 0.34 & 0.32 & 0.20 \\
\hline Embarrassing & 1.63 & 1.48 & 1.63 & 1.43 & 0.17 & 0.21 & 0.10 \\
\hline Vivid & 5.09 & 1.56 & 5.29 & 1.67 & 0.20 & 0.50 & 0.20 \\
\hline Unique & 3.97 & 1.99 & 4.00 & 1.94 & 0.03 & 0.35 & 0 \\
\hline
\end{tabular}

Note-All changes are unsigned, apart from vivid and unique, which incorporate direction of change. 
barrassment may be experienced only infrequently as an emotional response to a situation, so that few naturally occurring autobiographical memories actually represent it. There is some support for this assertion in the finding that the participants' ratings on this scale were uniformly low. Of the 210 initial ratings taken of embarrassment experienced in the retrieved episode, $89.5 \%$ were 2 or less on the 7 -point scale, with $80.1 \%$ being rated 1 , the lowest point on the scale. With respect to the personal scale, it can also be argued that, once retrieved, any memory will be experienced as personal, and additional details that are recovered will not affect the initial impression.

\section{EXPERIMENT 2}

In Experiment 1, the emotional aspects of a remembered episode were treated as one class of event detail. In Experiment 2, the issue is widened by asking whether the observed effect applies more generally to all types of event details. One of the rating scales used in the first experiment, vividness, suggests that this might be the case. However, the participants may well have felt that the memory was more vivid because their memory for the emotional aspects of the recalled episode, the main focus of the rating scales, was clearer. In measuring emotional versus other details, different approaches may be necessary. For emotional detail, quantification requires the measurement of emotional states remembered as having been experienced at the time of the episode. However, at the risk of stating the obvious, when we say that participants remember more emotion detail, this does not mean that they are remembering that a greater number of (different) emotions were experienced. Rather, they have access to a phenomenologically stronger record of the limited number of emotions that were experienced. This is why rating scales, rather than simple counts, are more appropriate for this type of event detail. In Experiment 2, more general event details were measured, using an approach that entailed transcribing the participants' recollections of events and comparing the numbers of new event details recalled after the second cues across different cue types.

An alternative explanation for the finding that congruent odors lead to enhanced recollection of event details relies on the introduction of a qualitatively different cue in the second retrieval phase, as opposed to use of the same, repeated cue in the label condition. Thus, there may be nothing special about odors; any cue that differs from the original cue and is congruent with the content of the recollected episode may produce the same result. To address this additive cue hypothesis, a visual cue condition, in which the participant was presented with a color photograph in the second retrieval phase, was included in Experiment 2. The photographs depicted, in each case, the source object of the label used as the first cue. This, then, represented a visual analogue of the congruent odor condition and was used to directly test the alternate hypothesis that any secondary cue that was congruent with a recollected episode would significantly enhance recall, relative to the label condition. In addition, the incongruent odor condition was retained as a control for the possibility that arousal levels may affect retrieval of event details.

\section{Method}

Participants. Forty volunteer participants, consisting of a mixture of undergraduate students and people in full-time employment, were assigned to one of four conditions (label, incongruent odor, visual, and congruent odor).

Materials. A reduced set of six odors was used: coffee, cinnamon, disinfectant, baby powder, paint, and cigarette. They were presented in a manner identical to that in Experiment 1 . In addition, $6 \times 4$ in. color photographs of each of the stimuli were used as cues in the visual cue condition.

Procedure. The participants were tested in quiet surroundings that were free of distractions and strong odors. All the participants were provided with the same instructions as those in Experiment 1. As a second cue in the extra visual condition, the participants were presented with a photograph of the stimulus given as a label. Each participant took part in three trials, and the same 3-min time limit was allowed for the recollective stage of each trial. The reports of the recollected events were tape recorded and transcribed for content analysis.

\section{Results and Discussion}

The contents of the recollected episodes were transcribed and content analyzed, using single sentences as the unit of analysis. Given that the source text was transcribed speech rather than written word and that normal speech tends not to involve strict discrete sentences, sentence form was imposed on the text by dividing long utterances into discrete sentence units when it seemed appropriate (i.e., when one sentence appeared to run into another). The mean number of sentences vocalized after the first and second cues in each condition were as follows (standard deviations in parentheses): label condition, Cue 1, 25.4 (13.2), Cue 2, 15.2 (11.1); visual condition, Cue 1, 24.6 (4.76), Cue 2, 9.6 (3.6); incongruent condition, Cue 1, 24.8 (6.9), Cue 2, 9.2 (7.2); congruent condition, Cue 1, 22.7 (8.1), Cue 2, 19.9 (11.6). The total number of sentences uttered after the first cue was tallied for each of the four conditions (label, 254; incongruent odor, 248; visual, 246; congruent odor, 227).

A chi-square analysis revealed no difference among the four conditions $\left(\chi^{2}=1.68\right.$, n.s. $)$, suggesting that the groups were evenly balanced in verbosity. The experimenter then rated the recollections taken after the second cue and assigned each sentence to one of two simple categories: new for sentences that contained information relating to the episode that did not appear previously in the recollec-

Table 2

Number of Sentences (Proportion of the Condition) in Each Content Analysis Coding Category and Total Number of Sentences From Each Condition

\begin{tabular}{lrllllr}
\hline & \multicolumn{2}{c}{ New } & & \multicolumn{2}{c}{ Irrelevant } & \\
\cline { 2 - 3 } Condition & No. & $P$ & & No. & $P$ & Total \\
\hline Label & 71 & .47 & & 81 & .53 & 152 \\
Incongruent & 33 & .36 & & 59 & .64 & 92 \\
Visual & 44 & .46 & & 52 & .54 & 96 \\
Congruent & 120 & 60 & & 79 & .40 & 199 \\
\hline
\end{tabular}


tion after the first cue, and irrelevant for sentences that did not contain new information (invariably, statements that did not contain new information were irrelevant to the retrieval of the episode). A subset of recollections was then independently analyzed by two blind raters (following Holsti, 1969), and the interrater reliability among all three was calculated (Krippendorff, 1980). The interrater agreement was found to be strong (Krippendorff's $\alpha=$ .717).

The number of sentences in each category from each condition is given in Table 2. As can be seen, the total numbers of sentences produced for the incongruent and the visual conditions were approximately matched (92 and 96, respectively), and were much lower than those observed for the label (152) and the congruent (199) conditions. For the latter two conditions, the difference in total numbers of sentences can be explained by the increases in the numbers of new details that emerged in Phase 2 (71 and 120, respectively), with the numbers of sentences containing irrelevant detail approximately matched (81 and 79, respectively). The relevant proportions of sentences containing new and irrelevant detail showed a similar pattern: For the congruent condition, the majority of sentences were those containing new detail; for the label and the visual conditions, the proportions were approximately matched; whereas for the incongruent condition, the majority of sentences were those containing irrelevant detail.

Given that the data took the form of frequency categorizations of a limited number of statements, chi-square analyses were deemed the most appropriate method of statistical analysis (Siegel \& Castellan, 1988). The data were subjected to a 2 (new vs. irrelevant) $\times 4$ (label vs. incongruent odor vs. visual vs. congruent odor) chi-square analysis, and the result proved to be significant $\left[\chi^{2}(3)=\right.$ $16.72, p<.001]$. Further partitioning of the $2 \times 4$ contingency table and subsequent partition chi-square analysis revealed that the congruent condition was significantly different from the other three conditions $\left[\chi^{2}=13.44, p<\right.$ $.001]$, indicating that episodes retrieved in response to the congruent odor cues included more new detail than did those retrieved in response to the label, picture, or incongruent odor cues.

These results provide clear support for the differential cue affordance value hypothesis. Retrieval was anchored to specific episodes in Phase 1, and the addition of a congruent olfactory cue in Phase 2 produced a significant increase in new details. That this effect resulted simply from the use of two different cues (the additive cue hypothesis) can be ruled out, because there was no evidence of a similar enhancement for the visual cue condition. Interestingly, the visual condition yielded a much lower total number of sentences than did the label condition. There are probably two (related) reasons for this. First, although not explicitly instructed to do so, the participants tended to focus on the visual details of the target cue as represented in their autobiographical recollection. In other words, the visual cues induced a selective search strategy. Second, in the majority of cases, the visual cues did not match those that fea- tured in the individuals' recollections, which led the participants to comment on the differences. One reason that the additive cue hypothesis was not supported here, therefore, may relate to the fact that the visual cues employed did not sufficiently map the visual appearance of the items in the recollective episode. Of course, exactly the same argument could be made about the (congruent) olfactory cues, because it is extremely unlikely that these matched exactly the sensory experiences of the original autobiographical episodes. Despite this, the overall proportion of new detail that emerged in the visual condition was no different from that in the label condition.

The inclusion of the incongruent odor condition in the present experiment permitted a test of one further hypothesis, that enhanced retrieval results from increased arousal levels associated with "sensing" odors. This hypothesis can also be ruled out, because there was no evidence of enhanced retrieval for the incongruent odor condition. In fact, as with the visual cues, there was evidence that, relative to the label condition, the total number of sentences produced in Phase 2 was attenuated. Also, although the visual and the incongruent label conditions led to approximately equivalent sentence production in Phase 2, the relative proportions of sentences containing new and irrelevant detail differed, with fewer new details being given in the incongruent odor condition. This echoes the pattern observed in Experiment 1, in which the change in ratings for the incongruent odor condition was less than that for the label condition on every scale (see Table 1 ). One reason for this may relate to the differential cue affordance hypothesis we set out to test. That is, odors are indeed especially potent as retrieval cues, to the extent that they automatically induce the retrieval of unrelated and irrelevant detail in the incongruent odor condition.

\section{GENERAL DISCUSSION}

Two sources of data, participants' ratings of their memory quality and content analysis of the recollective episodes recounted, provide converging evidence that odors are especially potent reminders of autobiographical experiences. Several plausible alternative hypotheses that might underlie the phenomenon were ruled out, through the use of the double-cuing methodology and specific control conditions. This permitted a test of what we believe represents the more popular interpretation of the Proust phenomenon, the differential cue affordance hypothesis, which we can now confidently say is unambiguously supported.

The findings reported here do not represent the only distinctive features of odor-cued memories that are relevant to the Proust phenomenon. As was noted in the introduction, odor-cued memories are postulated to be more detailed, more emotionally loaded, and more distant in time. Although the single-cue comparison method is unsuitable for testing particular hypotheses concerning the quantity and quality of detail retrieved, we have successfully applied the technique to evaluate the third of these memory attributes-namely, the age at which autobiographical 
episodes were originally experienced. In this case, the age of a memory, unlike quality or quantity, is a fixed property anchored in time and is therefore not open to bias. When the distribution of autobiographical memories across the entire lifespan in a group of elderly participants was plotted, we found that the peak number of memories retrieved (the so-called reminiscence bump; Rubin \& Schulkind, 1997) appeared at an earlier age for odor cues than the peak for label cues (Chu \& Downes, 2000). That is, odorcued autobiographical memories are older. Taken together, therefore, our experiments, employing two complementary methodologies, show that odor-cued memories tend to be more emotional, more detailed, and of a greater age. Thus, we can state with some confidence that the phenomenon attributed to Proust is more than literary speculation.

In common with the vast majority of studies in which autobiographical memories for personal events (rather than knowledge) are examined, our experiments present no means of assessing the accuracy of the details retrieved by our participants. The crux of Banaji and Crowder's (1989) controversial attack on the field of everyday memory involved a similar line of argument (see Conway, 1991, Morton, 1991, and Neisser, 1991, for rebuttals). One method of achieving the form of empirical control that Banaji and Crowder would demand (and one that we are currently pursuing in our laboratory) would be to arrange a series of naturalistic events for participants to experience. This series would involve a range of sensory elements (including odors) so that, after a long delay (i.e., months), detailed retrieval could be examined (and verified) in response to an array of different cue types. Still, we have no reason, in the present study, to presume that the proportion of inaccurate detail in one condition should differ from that in any other. Therefore, even allowing for the inaccuracy of a proportion of retrieved information, if a greater amount of absolute detail were to be retrieved in the congruent-odor condition, this would imply that a greater amount of accurate detail would be retrieved as well.

Why should odors differ from other cue types in these ways? One class of explanation draws on knowledge of the neuroanatomical projections to and from the olfactory cortex and the intrinsic links with circuits known to mediate episodic memory. Thus, the olfactory bulb projects to the amygdala, the fimbria of the hippocampus, and the dorso-medial nucleus of the thalamus (Dodd \& Castellucci, 1991; Nieuwenhuys, Voogd, \& van Huijzen, 1988), each of which has some involvement in memory consolidation processes (see Aggleton \& Brown, 1999; McGaugh, Roozendaal, \& Cahill, 2000). The work of McGaugh and colleagues, in particular, has shown that the amygdala plays a specific role in modulating hippocampal-mediated consolidation processes. Furthermore, in rats, this modulatory function is specifically related to appetitively or aversively motivated learning (McGaugh et al., 2000). Thus, the amygdala may be directly involved in the formation of emotional memories, a view supported by recent functional imaging studies of human participants (e.g., Hamman, Ely, Grafton, \& Kilts, 1999), and its modulatory role in memory consolidation will lead to the creation of more stable memory representations. Because of the neuroanatomical projections between olfactory processing regions and the limbic structures, odors may be especially privileged in their ability to directly influence the modulatory functions of the amygdala.

A second class of explanation is grounded in cognitive psychological theories of autobiographical memory retrieval. Conway's (1992, 1996; Conway \& Pleydell-Pearce, 2000) model of autobiographical memory structure proposes that autobiographical memory retrieval processes are cyclical in nature, so that, given a single cue word, memory probes become more refined and, thus, more specific with each retrieval cycle, eventually leading to the retrieval of details from an event-specific knowledge base. In contrast, highly specific cues, such as sensory stimuli, may be able to bypass the normal cyclic retrieval process and directly access the event-specific knowledge (ESK) base (Conway, 1992). The fact that there exist independent top-down (thematic) and bottom-up (sensory) retrieval processes, however, does not necessarily lead to the prediction that olfactory cues are selectively advantaged relative to other cue types (although it may predict that retrieval is faster for olfactory cues). This is because both provide access to the same pool of ESK. Possibly, the way in which the different types of cues map onto the knowledge base may differ for thematic and sensory information, so that the latter provides more diffuse links with event details. This is not to propose that ESK is organized by modality but, rather, that sensory cues differ from other cues in terms of access to the knowledge base. Nevertheless, without further independent specification, there is a danger of circularity in adding these speculative assumptions to the model.

Data from two experiments exploring naturally occurring olfactory-cued autobiographical memories have shown convergent support for the Proust phenomenon. In terms of the ratings of the emotional quality of odor-cued memories and the quantity of information contained in these recollections, odors are especially potent reminders of past experience.

\section{REFERENCES}

Aggleton, J. P., \& Brown, M. W. (1999). Episodic memory, amnesia, and the hippocampal-anterior thalamic axis. Behavioral \& Brain Sciences, 22, 425-489.

Baddeley, A. D. (1982). Domains of recollection. Psychological Review, 89, 708-729.

BANAJI, M. R., \& Crowder, R. G. (1989). The bankruptcy of everyday memory. American Psychologist, 44, 1185-1193.

BARon, R. A., \& Bronfen, M. I. (1994). A whiff of reality: Empirical evidence concerning the effects of fragrances on work-related behavior. Journal of Applied Social Psychology, 24, 1179-1203.

Chu, S., \& Downes, J. J. (2000). Long live Proust: The odour-cued autobiographical memory bump. Cognition, 75, B41-B50.

Clark, D. M., \& Teasdale, J. D. (1982). Diurnal variations in clinical depression and accessibility of memories of positive and negative experiences. Journal of Abnormal Psychology, 91, 87-95.

Conway, M. A. (1991). In defense of everyday memory. American Psychologist, 46, 19-26.

Conway, M. A. (1992). A structural model of autobiographical memory. 
In M. A. Conway, D. C. Rubin, H. Spinnler, \& W. A. Wagenaar (Eds.), Theoretical perspectives on autobiographicalmemory (pp. 167-193). Dordrecht: Kluwer.

Conway, M. A. (1996). Autobiographical knowledge and autobiographical memories. In D. C. Rubin(Ed.), Remembering ourpast: Studies in autobiographicalmemory (pp. 67-93). Cambridge: Cambridge University Press.

Conway, M. A., \& Pleydell-Pearce, C. W. (2000). The construction of autobiographical memories in the self-memory system. Psychological Review, 107, 261-288.

Dodd, J., \& Castellucci, V. F. (1991). Smell and taste: The chemical senses. In E. R. Kandel, J. H. Schwartz, \& T. M. Jessell (Eds.), Principles of neural science (3rd ed., pp.512-529). Norwalk: Appleton Lange.

Ehrlichman, H., \& Bastone, L. (1992). Olfaction and emotion. In M. J. Serby \& K. L. Chobor(Eds.), Science of olfaction (pp. 410-438). New York: Springer-Verlag.

EHRLICHMAN, H., \& HALPERN, J. N. (1988). Affect and memory: Effects of pleasant and unpleasant odor on retrieval of happy and unhappy memories. Journal of Personality \& Social Psychology, 55, 769-779.

Engen, T., \& Ross, B. M. (1973). Long-term memory of odors with and without verbal descriptors. Journal of Experimental Psychology, 100, 221-227.

Hamman, S. B., Ely, T. D., Grafton, S. T., \& Kilts, C. D. (1999). Amygdala activity related to enhanced memory for pleasant and aversive stimuli. Nature Neuroscience, 2, 289-293.

Herz, R. S. (1996). A comparison of olfactory, visual and tactile cues for emotional and non-emotional associated memories. Chemical Senses, 21, 614-615.

Herz, R. S., \& CuPChIK, G. C. (1992). An experimental characterization of odor-evoked memories in humans. Chemical Senses, 17, 519-528.

Herz, R. S., \& CupchiK, G. C. (1995). The emotional distinctiveness of odor-evoked memories. Chemical Senses, 20, 517-528.

Herz, R. S., \& ENGEN, T. (1996). Odor memory: Review and analysis. Psychonomic Bulletin \& Review, 3, 300-313.

Holsti, O. R. (1969). Content analysis for the social sciences and humanities. Reading, MA: Addison-Wesley.

KRIPPENDORFF, K. (1980). Content analysis: An introductionto its methodology. Beverly Hills, CA: Sage.

Lehrner, J. P., Walla, P., Laska, M., \& Deecke, L. (1999). Different forms of human odor memory: A developmental study. Neuroscience Letters, 272, 17-20.
LEVINE, L. J. (1997). Reconstructing memory for emotions. Journal of Experimental Psychology: General, 126, 165-177.

Lorig, T. S., \& SchwarTz, G. E. (1988). Brain and odor: I. Alteration of human EEG by odor administration. Psychobiology, 16, 281-284.

McGaugh, J. L., RoozendaAl, B., \& Cahill, L. (2000). Modulation of memory storage by stress hormones and the amygdaloid complex. In M. S. Gazzaniga (Ed.), The new cognitive neurosciences (2nd ed., pp. 1081-1098). Cambridge, MA: MIT Press.

Morton, J. (1991). The bankruptcy of everyday thinking. American Psychologist, 46, 32-33.

NeISSER, U. (1991). A case of misplaced nostalgia. American Psychologist, 46, 34-36.

Nieuwenhuys, R, Voogd, J., \& VAn HuiJen, C. (1988). The human central nervous system: A synopsis and atlas. New York: Springer-Verlag.

Proust, M. (1960). Swann's way. (C. K. Scott Moncrieff, Trans.). London: Chatto \& Windus. (Original work published 1922)

Roberts, A., \& Williams, J. M. G. (1992). Effects of olfactory stimulation on fluency, vividness of imagery and associated mood. British Journal of Medical Psychology, 65, 197-199.

Rubin, D. C., Groth, E., \& Goldsmith, D. J. (1984). Olfactory cuing of autobiographical memory. American Journal of Psychology, 97, 493-507.

Rubin, D. C., \& Schulkind, M. D. (1997). The distribution of autobiographical memories across the lifespan. Memory \& Cognition, 25, 859-866.

Schaв, F. R. (1991). Odor memory: Taking stock. Psychological Bulletin, 109, 242-251.

Siegel, S., \& CASTEllan, N. J. (1988). Nonparametric statistics for the behavioral sciences. (2nd ed.). New York: McGraw-Hill.

Tulving, E., \& Bower, G. H. (1974). The logic of memory representations. In G. H. Bower (Ed.), The psychology of learning and motivation: Advances in research and theory (Vol. 8, pp. 265-301). London: Academic Press.

White, T. L. (1998). Olfactory memory: The long and the short of it. Chemical Senses, 23, 433-441.

(Manuscript received July 17, 2000; revision accepted for publication January 23, 2002.) 